\title{
Stabilities of one-dimensional stationary states of Bose-Einstein condensates
}

\author{
Wenhua Hai ${ }^{1 *}$ Guishu Chong ${ }^{1,2}$, Jianwen Song ${ }^{1}$ \\ ${ }^{1}$ Department of Physics, Hunan Normal University, and \\ Key Laboratory of Quantum Structure and Manipulation of \\ Ministry of Education, Changsha 410081, China \\ ${ }^{2}$ Department of physics, Hunan University, Changsha 410082, China
}

\begin{abstract}
We explore the dynamical stabilities of a quasi-one dimensional (1D) Bose-Einstein condensate (BEC) consisting of fixed $N$ atoms with time-independent external potential. For the stationary states with zero flow density the general solution of the perturbed time evolution equation is constructed, and the stability criterions concerning the initial conditions and system parameters are established. Taking the lattice potential case as an example, the stability and instability regions on the parameter space are found. The results suggest a method for selecting experimental parameters and adjusting initial conditions to suppress the instabilities.
\end{abstract}

Keywords: Bose-Einstein condensate, Lyapunov stability, stability criterion, stability region, lattice potential

PACS numbers: 03.75.Kk, 32.80.Pj, 03.75.Lm, 05.45.-a

\section{Introduction}

Experimental observation of atomic gas Bose-Einstein condensates (BECs) has caused significant stimulation to the study of macroscopic quantum phenomena with nonlinearity. In the

${ }^{*}$ Author to whom all correspondence should be addressed, Email address: whhai2005@yahoo.com.cn 
mean field regime where the BECs are governed by the Gross-Pitaevskii equations (GPE), the BEC of a stationary state can be observed carefully in experiments only for the stable solutions of GPE. For the purpose of applications, the studies on the stability and instability of the solutions of GPE are necessary and important [1]-[17]. Recently, the instabilities of BECs have attracted much interest and the corresponding experimental [1, 2, 3] and theoretical [4]-[13] works were reported for various BEC systems. Several different definitions such as the Landau instability [5, 6], dynamical instability [11], quantum instability [12], parametric instability [9] and modulational instability [18] were employed. The used research methods concerned the characteristic exponent technique [4], Gaussian variational approach [7], and the numerical simulations to the partial differential equations [5, 11, 10]. The reported results showed that the instabilities are associated with the BEC collapse [18, 19], implosion and chaos [20] - [26], dynamical superfluid-insulator transition [27], and the formation and evolution of the matterwave bright solitons [28, 29, 30]. In order to stabilize the BECs [17], some stability criteria [15] and parameter regions [4, 5, 31, 32] were demonstrated. Most of the works focus in the stabilities under random perturbations. Experimentally [3] and theoretically [5] investigating the stabilities under the controllable perturbations has also become a challenging problem.

In the sense of Lyapunov, the instability entails that the initially small deviations from the unperturbed state grow without upper limit. We shall restrict the dynamical instability to the particular case of nonzero characteristic exponents such that the minor deviations from the unperturbed state grow exponentially fast [5, 11]. All of the above-mentioned investigations on the dynamical stabilities and instabilities are based on such a type of instability. By the control of instability we mean to induce the transitions from unstable states to stable ones. Realization of the control needs selecting the system parameters to enter the stability regions, or initially using a controllable perturbation as a control signal to suppress the growth of perturbed solutions. Any experiment always contains a degree of noise, that leads to the random perturbations to the system. Therefore, in order to suppress the known unstable motions, we have to initially adjust the system by using the control signal being stronger than the noise.

In the previous work, we have investigated the stabilities of BECs for the time-dependent chaotic states [23, 25] and dissipative cases [31]. In this paper, we shall consider the dynamical stability of the stationary states for a quasi-1D BEC consisting of fixed $N$ atoms with time- 
independent external potential and atomic scattering length. It will be demonstrated that for the case of zero flow density the bounded perturbed solutions depend on the external potential, condensed atom number, and the initial disturbances. The dependence implies that the stationary state of BEC is certainly stable only for the given parameter region and the possible instability can be suppressed by some initial adjustments. We take the BECs held in an optical lattice as an exemplification to illustrate the results on the stability, instability and undetermined stability. The results contain the known analytical assertions for the optical potential case [5, 11] and supply a method for selecting experimental parameters and adjusting initial conditions to establish the stable motions of BEC.

\section{Linearized equations and their solutions in the case of zero flow density}

We start with the dimensionless quasi-1D GPE [11, 33, 34]

$$
i \psi_{t}=-\frac{1}{2} \psi_{x x}+\left[V(x)+g_{1}|\psi|^{2}\right] \psi
$$

where the suitable units with $\hbar=m=1$ have been considered, $V(x)$ denotes the external potential, the quasi-1D interaction intensity $g_{1}$ is related to the $s$-wave scattering length $a_{s}$, atomic mass $m$ and the transverse trap frequency $\omega_{r}$ [35, 36] for the normalized wave-function $\psi$ with norm $|\psi|^{2}$ being the linear density of atomic number [11, 34]. It is well known that different solutions of a nonlinear equation may possess different stabilities. Here we study stability only for the stationary state solution of the form

$$
\psi_{0}=R(x) \exp [i \theta(x)-i \mu t]
$$

where $\mu$ is the chemical potential, $R(x)$ and $\theta(x)$ represent the module and phase, which are both the real functions. In the considered units, the phase gradient $\theta_{x}$ is equal to the flow velocity field. Given the module, we define the useful Hermitian operators [11]

$$
L_{n}=-\frac{1}{2} \frac{\partial^{2}}{\partial x^{2}}+n g_{1} R^{2}+V(x)-\mu, \quad \text { for } n=1,3 .
$$

Then inserting Eq. (2) into Eq. (1) gives the equations

$$
L_{1} R(x)=0
$$


In the equation we have assumed the flow velocity field and current density being zero.

We now investigate the stability of stationary state Eq. (2) by using the linear stability analysis, which is associated with boundedness of the perturbed solution [11, 15]

$$
\psi=\left[R(x)+\varepsilon \phi_{1}(x, t)+i \varepsilon \phi_{2}(x, t)\right] \exp [i \theta(x)-i \mu t]
$$

where the perturbed correction $\varepsilon \phi_{i}(x, t)$ is real function with constant $|\varepsilon| \ll 1$. Substituting Eqs. (5) and (4) into Eq. (1) yields the linearized equations [11]

$$
\phi_{1 t}=L_{1} \phi_{2}, \quad \phi_{2 t}=-L_{3} \phi_{1}
$$

For most of external potentials $V(x)$ we cannot derive the exact solutions from Eq. (1) or Eq. (4) such that the operators $L_{n}$ cannot be determined exactly. In the case of optic lattice potential, some specially exact solutions have been found [11, 10, 36], however, solving Eq. (6) for the general solution is still difficult. Therefore, we have to focus our attentions to the dynamical stability which is associated with the perturbed solutions of space-time separation,

$$
\phi_{i}(x, t)=T_{i}(t) \varphi_{i}(x), \quad \text { for } \quad i=1,2 .
$$

Note that the real function $\phi_{i}$ limits $T_{i}$ and $\varphi_{i}$ to real or imaginary simultaneously, the difference between both is only a sign "-" of $\phi_{i}$. We take real $T_{1}, \varphi_{1}, T_{2}$, and $\varphi_{2}$ without loss of generality, since the changes of the signs of $\phi_{i}$ do not affect the stability analysis. We shall discuss how to establish the sufficient conditions of stability as follows.

Combining Eq. (6) with Eq. (7), we get the coupled ordinary differential equations

$$
\begin{aligned}
\dot{T}_{1}(t) & =\lambda_{1} T_{2}(t), \quad \dot{T}_{2}(t)=-\lambda_{2} T_{1}(t) \\
L_{3} \varphi_{1}(x) & =\lambda_{2} \varphi_{2}(x), \quad L_{1} \varphi_{2}(x)=\lambda_{1} \varphi_{1}(x) .
\end{aligned}
$$

Here $\lambda_{i}$ is the real eigenvalue determined by the initial perturbations $\dot{T}_{i}(0), T_{i}(0)$. The corresponding decoupled equations are derived easily from the coupled ones as

$$
\begin{aligned}
& \ddot{T}_{i}(t)=-\lambda_{1} \lambda_{2} T_{i}(t), \lambda_{1}=\frac{\dot{T}_{1}(0)}{T_{2}(0)}, \lambda_{2}=-\frac{\dot{T}_{2}(0)}{T_{1}(0)} \\
& L_{1} L_{3} \varphi_{1}=\lambda_{1} \lambda_{2} \varphi_{1}, \quad L_{3} L_{1} \varphi_{2}=\lambda_{1} \lambda_{2} \varphi_{2} .
\end{aligned}
$$

Obviously, the general solutions of Eq. (10) can be written as the exponential functions

$$
\begin{gathered}
T_{i}=A_{i} e^{\lambda t}+B_{i} e^{-\lambda t}, \quad \lambda=\sqrt{-\lambda_{1} \lambda_{2}}, \\
A_{i}=\frac{1}{2}\left[T_{i}(0)+\frac{1}{\lambda} \dot{T}_{i}(0)\right], \quad B_{i}=\frac{1}{2}\left[T_{i}(0)-\frac{1}{\lambda} \dot{T}_{i}(0)\right],
\end{gathered}
$$


where $A_{i}, B_{i}$ are real or complex constants, which make $T_{i}(t)$ the real functions. Based on the existence of bounded eigenstates $\varphi_{i}(x)$, the results are classified as the three cases:

(i) Stability criterion: The eigenstates of Eq. (11) are bounded if and only if their eigenvalues are positive, $\lambda_{1} \lambda_{2}=-\lambda^{2}>0$, that makes $\lambda$ the imaginary constant and $T_{i}$ the periodic functions.

(ii) Instability criterion: One can find a negative eigenvalue $\lambda_{1} \lambda_{2}=-\lambda^{2}<0$ associated with a set of bounded eigenstates of Eq. (11) that makes $T_{i}$ the real exponential function.

(iii) Undetermined stability: One cannot determine whether all eigenvalues of the bounded eigenstates of Eq. (11) are positive. In this case, we can use criterion (i) to control the possible instability of case (ii).

\section{Stability regions on the parameter space and control of instability}

It is interesting noting that if the initial perturbations can be determined, the dynamical instability of real $\lambda$ case can be controlled by adjusting the initial disturbances to obey $A_{i}=0$ that will suppress the exponentially rapid growth of $T_{i}$ in Eq. (12). From Eqs. (12) and (10) we establish the controlling criteria for the instability as $\dot{T}_{i}(0)=-\lambda T_{i}(0)$. However, for the random initial perturbations such a control is difficult to do, since we cannot determine the initial values $\dot{T}_{i}(0)$ and $T_{i}(0)$. Therefore, in the case of random perturbation we are interested in determining the same eigenvalue $\lambda_{1} \lambda_{2}$ of operators $L_{1} L_{3}$ and $L_{3} L_{1}$, since the stability can be established if and only if the eigenvalue is positive such that $\lambda^{2}=-\lambda_{1} \lambda_{2}<0$. Let $\alpha \geq \alpha_{g}$ and $\beta \geq \beta_{g}$ be the eigenvalues of operators $L_{1}$ and $L_{3}$, which are determined by the eigenequations $L_{1} u(x)=\alpha u(x), \quad L_{3} v(x)=\beta v(x)$ with $u$ and $v$ being their eigenfunctions, where $\alpha_{g}$ and $\beta_{g}$ express the corresponding ground state eigenvalues respectively. From Eq. (3) we know the relation $L_{3}=L_{1}+2 g_{1} R^{2}$ that means $\alpha_{g}<\beta_{g}$ for $g_{1}>0$ and $\alpha_{g}>\beta_{g}$ for $g_{1}<0$. It is clear that Eq. (4) is one of the eigenequations of $L_{1}$ for the eigenvalue $\alpha=0$ so that the ground state eigenvalue obeys $\alpha_{g} \leq 0$ for any $g_{1}$. Then $\beta_{g}$ can be positive or negative for $g_{1}>0$ and $\beta_{g}<0$ for $g_{1}<0$.

From the above-mentioned results we establish the stability and instability conditions: 
Case $g_{1}>0$ : The sufficient condition of stability is $\alpha_{g}=0$, since such a ground state eigenvalue implies $\alpha \geq 0$ and $\beta>0$ for all of the eigenstates such that the well known spectral theorem gives [11, 10, 37] $\lambda_{1} \lambda_{2} \geq 0$. The corresponding sufficient conditions of instability reads $\alpha_{g}<0$ and $\beta_{g}>0$.

Case $g_{1}<0$ : The ground state eigenvalues satisfy the inequality $\beta_{g}<\alpha_{g} \leq 0$. So the sufficient condition of instability is $\alpha_{g}=0$.

In all the other cases, we don't know whether $\lambda_{1} \lambda_{2}$ is certainly positive or negative, so the linear stabilities are analytically undetermined. It is worth noting that Eq. (4) infers $R(x)$ to be one of the eigenstates of $L_{1}$ with eigenvalue $\alpha_{R}=0$. Therefore, if $R(x)$ is a ground state, the above stability and instability conditions indicate that this state is stable for $g_{1}>0$, and unstable (or metastable) for $g_{1}<0$.

Note that all the above-mentioned results are valid for arbitrary time-independent potential. We will take the BEC held in an optical lattice as a concrete physical example to evidence these results. In the lattice potential case, the above-mentioned sufficient conditions agree with the stability and instability criterions established by the authors of Ref. [11]. We shall apply the sufficient stability and instability conditions to find the corresponding stability and instability regions on the parameter space, and apply these results to study the stabilization of the considered BEC system.

For an arbitrary time-independent potential, the eigenequation $L_{1} u=\alpha u$ can be rewritten as the integral form 38 ]

$$
\begin{aligned}
u & =u_{1}+u_{2} \\
u_{1} & =q^{-1} e^{-q x} \int e^{q x} f u d x, u_{2}=-q^{-1} e^{q x} \int e^{-q x} f u d x \\
f & =q^{2} / 2+\alpha+\mu-V(x)-g_{1} R^{2}(x)
\end{aligned}
$$

where $q>0$ is a real constant. This integral equation can be directly proved by taking the second derivative from its both sides. The integrals in Eq. (13) are indefinite, what means that the solutions are defined with accuracy of two additive constants. While the eigenequation $L_{1} u=\alpha u$ just is a second order equation which also implies two arbitrary constants determined by the boundary conditions. It is the two additive constants to make the integral equation (13) completely equivalent to the eigenequation. The stability requires the eigenstate to be bounded and the possible bounded solution $u$ must satisfy the boundedness condition 
$\lim _{x \rightarrow \pm \infty} \int e^{\mp q x} f u d x=0$. Under this condition and for the lattice potential case, we can apply the l'Höpital rule to get the superior limit [25]

$$
\varlimsup_{x \rightarrow \pm \infty} u \leq \varlimsup_{x \rightarrow \pm \infty} u_{1}+\varlimsup_{x \rightarrow \pm \infty} u_{2}=2 q^{-2} \overline{\lim }_{x \rightarrow \pm \infty}(f u)
$$

Note that there is not the usual limit, because of the periodicity of lattice potential. It is clear that the solution of linear equation $L_{1} u=\alpha u$ can be taken as $u(x)=A u^{\prime}(x)$ with arbitrary constant $A$ and any solution $u^{\prime}(x)$ such that one can always select $u$ to obey $\overline{\lim }_{x \rightarrow \pm \infty} u>0$. Thus Eq. (14) implies $2 q^{-2} \overline{\lim }_{x \rightarrow \pm \infty} f \geq 1$, namely

$$
\alpha \geq-\left\{\mu+\varlimsup_{x \rightarrow \pm \infty}\left[-V(x)-g_{1} R^{2}(x)\right]\right\}=\alpha_{g}
$$

For the eigenequation $L_{3} v=\beta v$ after using $3 g_{1}$ instead of $g_{1}$, the same calculations give

$$
\beta \geq-\left\{\mu+\varlimsup_{x \rightarrow \pm \infty}\left[-V(x)-3 g_{1} R^{2}(x)\right]\right\}=\beta_{g}
$$

Combining Eq. (15) with the stability sufficient condition [11] $\alpha_{g}=0$ for $g_{1}>0$, we get the parameter region of stability

$$
\mu=\mu_{s}=-\varlimsup_{x \rightarrow \pm \infty}\left[-V(x)-g_{1} R^{2}(x)\right] \text { for } g_{1}>0
$$

which contains the relation among $\mu, g_{1}$ and the potential parameters. Applying Eqs. (15) and (16) to the instability sufficient conditions [11] $\alpha_{g}<0, \beta_{g}>0$ for $g_{1}>0$ and $\alpha_{g}=0$ for $g_{1}<0$, we get the parameter regions of instability

$$
\begin{gathered}
-\varlimsup_{x \rightarrow \pm \infty}\left[-V(x)-g_{1} R^{2}(x)\right]<\mu=\mu_{i n}<-\overline{\lim }_{x \rightarrow \pm \infty}\left[-V(x)-3 g_{1} R^{2}(x)\right] \text { for } g_{1}>0 \\
\mu_{i n}=-\overline{\lim }_{x \rightarrow \pm \infty}\left[-V(x)-g_{1} R^{2}(x)\right] \text { for } g_{1}<0 .
\end{gathered}
$$

By the sufficient conditions we mean that the stationary state $R(x) e^{-i \mu t}$ of Eq. (1) is certainly stable for the $\mu$ values in the parameter region fixed by Eq. (17), and the stationary states are certainly unstable for the $\mu$ values in any region of Eq. (18). The dynamical stabilities are undetermined outside.

We now see the physical meaning of the stability relation in Eq. (17) for the stationary states of BEC with zero current density. Setting the sum of external potential and internal interaction as $U(x)=V(x)+g_{1} R^{2}(x)$ with periodic $V(x)$ and bounded $R(x)$, when $U(x) \geq B$ is satisfied for all $x$ values and a fixed constant $B$, Eq. (17) implies $\mu_{s}=B \leq U(x)$. Namely the sufficient 
stability condition means that if the chemical potential is equal to the minimum of $U(x)$, the considered states are certainly stable. For a known state the stability can be easily examined by using Eq. (17). We have tested the exact solutions given in Ref. [11] for the potential $V(x)=$ $-V_{0} \operatorname{sn}^{2}(x, k)$ and found that some of them have the instabilities and undetermined stabilities, where $\left|V_{0}\right|$ is the potential depth and $\operatorname{sn}(x, k)$ the Jacobian elliptic sine function with $k$ being the modulus. Substituting one of the exact solutions, $g_{1} R^{2}(x)=-\left(1+V_{0} / k^{2}\right)\left[1-k^{2} s n^{2}(x, k)\right]$ with the potential depth $-V_{0} \geq k^{2}$ and chemical potential $\mu=-1-V_{0} / k^{2}+k^{2} / 2$ [see Eq. (12) of Phys. Rev. E63, 036612(2001)], into Eq. (17) yields the stability parameter relation $\mu_{s}=-1-V_{0} / k^{2}$. A difference of $k^{2} / 2$ exists between the $\mu_{s}$ value required by the stability condition and the chemical potential $\mu$ in the exact solution, namely the stability criterion (17) is not met here. This assertion differs from the result of Ref. [11], where this solution fits their stability criterion and the stability is independent of the parameters $k$ and $V_{0}$. However, when the potential depth $\left|V_{0}\right|$ is much greater than the modulus $k$ (e.g. $V_{0}=-1$ and $k=0.2$ ), we have the chemical potential near the stability relation $(17)\left(\mu=24.02=\mu_{s}+0.02 \approx \mu_{s}\right)$. This infers the higher stability being associated with a smaller value of the modulus $k$ and a relatively greater $\left|V_{0}\right|$ value. Thus our stability parameter criterion suggests that for a known solution with instability or undetermined stability one can raise the practical stability by adjusting the system parameter (e.g. the above $k$ and $\left|V_{0}\right|$ ) to approach the values of the stability region in Eq. (17).

Generally, constructing a stable exact solution of GPE is not easy, because of the nonintegrability of Eq. (4) with periodic potential. However, in the large- $N$ limit, we can fulfil the criterion (17) for the case of a repulsive nonlinearity, since the Thomas-Fermi (TF) approximation [33] $U(x)=\mu_{T F}$ just fits the stability relation. Therefore, it is practical relevant to prepare such a stable TF state $R\left(x, \mu_{T F}\right)$ by increasing the condensed atom number $N$. Given the number $N$ and the periodic boundary condition experimentally, from the normalization condition $N=n \int_{0}^{\pi} R^{2}\left(x, \mu_{T F}\right) d x=n \int_{0}^{\pi}\left[\mu_{T F}-V(x)\right] d x / g_{1}$ we derive the chemical potential of the stable TF state

$$
\mu_{T F}=\mu_{s}=\frac{N g_{1}}{n \pi}+\frac{1}{\pi} \int_{0}^{\pi} V(x) d x
$$

which is related to the atom number $N$ and the potential strength $V_{0}$ and period $K(k)$, where $n \sim 100$ is the lattice number. In fact, noticing the dependence of $R=R(x, \mu)$ on $\mu$ in Eq. (4), 
the normalization condition of any known state can also lead to $\mu=\mu(N)$ and $R=R(x, N)$. Applying them to eliminate $\mu$ in Eqs. (17) and (18) will give the corresponding relationships among the experimental parameters $N, g_{1}, V_{0}$ and $K(k)$. So we can control the instability of the known state by selecting the experimental parameters to fit or to approach the stability region of Eq. (17). In many practical cases, we cannot obtain the exact solution of Eq. (4) for some periodic potentials, that necessitates the numerical investigation. In order to fit (or near) the stability region in Eq. (17) and to avoid the instability regions of Eq. (18), we could use Eq. (19) to estimate and adjusted the chemical potential in region $\mu \approx \mu_{T F}$ such that the stability of the numerical solutions of Eq. (4) can also be established or improved.

On the other hand, in the case of arbitrary time-independent potential, for some known unstable solutions $R=R\left(x, \mu_{i n}\right)$ from Eqs. (10) and (12) we can experimentally set and adjust the initially controllable perturbation as a control signal [3] to suppress the exponentially fast growth of $T_{i}(t)$. Although the phase $\theta$ and amplitude $R$ are time-independent in the considered case, the initial perturbations can result in the nontrivial and time-dependent corrections to the phase and atomic-number density. From Eq. (5) we find their first corrections as

$$
\begin{aligned}
& \triangle \theta(x, t) \approx \arctan \left[\varepsilon T_{2}(t) \varphi_{2}(x) / R(x)\right] \approx \varepsilon T_{2}(t) \varphi_{2}(x) / R(x), \\
& \triangle|\psi|^{2}(x, t) \approx 2 \varepsilon T_{1}(t) \varphi_{1}(x) R(x)
\end{aligned}
$$

which are initially proportional to $T_{1}(0)$ and $T_{2}(0)$ respectively. Making use of Eq. (20), the adjustments to the initially controllable perturbations can be performed by trimming the number density $|\psi|^{2}$, velocity field $(\triangle \theta)_{x}$ and their time derivatives which are proportional to the corresponding trimming velocities. Given Eqs. (10) and (12), we know the stability initial criterion

$$
\lambda^{2}=-\lambda_{1} \lambda_{2}=\dot{T}_{1}(0) \dot{T}_{2}(0) /\left[T_{1}(0) T_{2}(0)\right]<0
$$

Once Eq. (21) is satisfied in the adjustments to the initial perturbations, Eq. (12) becomes the periodic solution which implies the stability. Although we cannot determine the initial values $\dot{T}_{i}(0)$ and $T_{i}(0)$, experimentally, the number density can be adjusted by varying the condensed atom number, and the adjustments to superfluid velocity may be related to a displacement $\triangle x$ of a magnetic potential [3]. According to Eqs. (20) and (21), if we initially increases (or decreases) both the relative derivative $\dot{T}_{2}(0) / T_{2}(0)=\left.\frac{\partial \triangle \theta_{x}(x, t)}{\partial t}\right|_{t=0} / \triangle \theta_{x}(x, 0)$ of flow velocity and 
the relative derivative $\dot{T}_{1}(0) / T_{1}(0)=\left.\frac{\partial \triangle|\psi|^{2}(x, t)}{\partial t}\right|_{t=0} / \triangle|\psi|^{2}(x, 0)$ of atomic number density, the stability initial criterion (21) is destroyed and the system will become unstable. But when one of them is increased and another is decreased simultaneously, the stability criterion (21) is satisfied and the possible instability is suppressed. These assertions may be tasted experimentally.

\section{Conclusions and discussions}

In conclusion, we have investigated the dynamical stability, instability and undetermined stability of a quasi-1D BEC in the stationary states for time-independent external potential and atomic scattering length, and fixed atomic number. After space-time separation of variables, we derive the general solutions of the linearized time-evolution equations for the trivial phase case and give a stability criterion related to the initial conditions. As an important example, we evidence the stability criterion analytically for the BEC held in an optical lattice potential. By using the known sufficient conditions of stability and instability [11], several parameter regions of stability and instability are shown. Our results contain some new stability predictions which can be tested with current experimental setups. Finally, we stress that applying our stability initial criterion and parameter region one can stabilize the considered BEC system by adjusting the system parameters experimentally to enter or near the stability region of Eq. (17) on the parameter space. For the parameters out of the stability region we can also establish or improve the stability by adjusting the initial flow velocity and atomic number density to fit or approach the stability initial criterion.

Acknowledgment This work was supported by the National Natural Science Foundation of China under Grant No. 10575034 and by the Key Laboratory of Magnetic Resonance and Atomic and Molecular Physics of China under Grant No. T152504.

\section{References}

[1] J. K. Chin, J.M. Vogels, and W. Ketterle, Phys. Rev. Lett. 90, 160405 (2003).

[2] L. Fallani, L. De Sarlo, J. E. Lye, M. Modugno, R. Saers, C. Fort, and M. Inguscio, Phys. Rev. Lett., 93, 140406(2004). 
[3] S. Burger, F.S. Cataliotti, C. Fort, F. Minardi, M. Inguscio, M.L. Chiofalo, and M.P. Tosi, Phys. Rev. Lett. 86, 4447 (2001).

[4] Y. Zheng, M. Koštrun, and J. Javanainen, Phys. Rev. Lett. 93, 230401 (2004).

[5] B. Wu and Q. Niu, Phys. Rev. A 64, 061603(R) (2001).

[6] M. Machholm, C. J. Pethick, and H. Smith, Phys. Rev. A 67, 053613 (2003).

[7] F. Kh. Abdullaev, A. Gammal, Lauro Tomio, and T. Frederico, Phys. Rev. A 63, 043604(2001).

[8] J.D. Carter and H. Segur, Phys. Rev. E 68, 045601(2003).

[9] G. M. Genkin, Phys. Rev. A 63, 025602(2001).

[10] B. Deconinck, J N. Kutz, M. S Patterson and B. W Warner, J. Phys. A: Math. Gen. 36, $5431(2003)$.

[11] J.C. Bronski, L.D. Carr, B. Deconinck, and J.N. Kutz, Phys. Rev. Lett. 86, 1402(2001); J. C. Bronski, L. D. Carr, B. Deconinck, J. N. Kutz, and K. Promislow, Phys. Rev. E63, 036612(2001); L. D. Carr, J. N. Kutz and W. P. Reinhardt, Phys. Rev. E63, 066604(2001); J. C. Bronski, L. D. Carr, R. Carretero-Gonzalez, B. Deconinck, J. N. Kutz, and K. Promislow, Phys. Rev. E64, 056615(2001).

[12] V. S. Shchesnovich, Phys. Lett. A349, 398(2006).

[13] F. Abdullaev, A. Abdumalikov and R. Galimzyanov, Phys. Lett. A367, 149(2007).

[14] A. Trombettoni and A. Smerzi, Phys. Rev. Lett. 86, 2353(2001).

[15] L. Bergé, T. J. Alexander, and Yuri S. Kivshar, Phys. Rev. A 62, 023607(2000).

[16] J-K Xue, G-Q Li and P Peng, Phys. Lett. A358, 74(2006).

[17] H. Saito and M. Ueda, Phys. Rev. Lett. 90, 040403 (2003)

[18] V. V. Konotop and M. Salerno, Phys. Rev. A 65, 021602(R)(2002).

[19] Y. Kagan, A. E. Muryshev, and G. V. Shlyapnikov, Phys. Rev. Lett. 81, 933(1998). 
[20] H. Saito, Ueda M. Phys. Rev. Lett. 86, 1406(2001).

[21] F. Kh. Abdullaev and R. A. Kraenkel, Phys. Rev. A 62, 023613 (2000).

[22] C. Lee, W. Hai, L. Shi, X. Zhu, and K. Gao, Phys. Rev. A 64, 053604(2001).

[23] W. Hai, C. Lee, G. Chong and L. Shi, Phys. Rev. E 66, 026202(2002).

[24] G. P. Berman and F. M. Izrailev, Chaos, 15, 015104 (2005).

[25] G. Chong, W. Hai and Q. Xie, Chaos 14, 217(2004); Phys. Rev. E 70, 036213 (2004).

[26] B. Xia, W. Hai and G. Chong, Phys. Lett. A351, 136(2006).

[27] A. Smerzi, A. Trombettoni, P. G. Kevrekidis, and A. R. Bishop, Phys. Rev. Lett. 89, $170402(2002)$.

[28] K.E. Strecker, G.B. Partridge, A.G. Truscott and R.G. Hulet, Nature, 417, 150(2002).

[29] L. D. Carr, and J. Brand, Phys. Rev. Lett., 92, 040401(2004).

[30] L. Salasnich, A. Parola, and L. Reatto, Phys. Rev. Lett., 91, 080405(2003).

[31] X. Luo and W. Hai, Chaos 15, 033702(2005).

[32] A. Montina, R. Mannella and E. Arimondo, Phys. Lett. A261, 337(1999).

[33] F. Dalfovo, S. Giorgini, L.P. Pitaevskii and S. Stringari, Rev. Mod. Phys. 71, 463(1999).

[34] A. J. Leggett, Rev. Mod. Phys. 73, 307(2001).

[35] S.A. Gardiner, D. Jaksch, R. Dum, J.I. Cirac, P. Zoller, Phys. Rev. A 62, 023612 (2000).

[36] W. Hai, C. Lee, X. Fang and K. Gao, Physica A335, 445(2004).

[37] R. Courant and D. Hilbert, Methods of Mathematical Physics (Wiley, New York, 1989).

[38] W. Hai, M. Feng, X. Zhu, L. Shi, K. Gao, and X. Fang, Phys. Rev. A 61, 052105(2000). 